\title{
Humic substances of peloid as natural adaptogens
}

\author{
Avvakumova N.P. ${ }^{1}$, Shurygina O.V. ${ }^{2}$, Krivopalova M.A. ${ }^{1}$, Rusakov D.A. ${ }^{2}$, Zhernov Yu.V. ${ }^{3}$, \\ Glubokova M.N. ${ }^{1}$, Katunina E.E. ${ }^{1}$ \\ ${ }^{1}$ Samara State Medical University, Samara, Russia \\ 2IDK Medical Company - MD Medical Group "Mother and Child", Moscow, Russia \\ ${ }^{3}$ National Research Center - Institute of Immunology FMBA of Russia, Moscow, Russia \\ doi: 10.36291/HIT.2019.avvakumova.087
}

Today the fact that the mobility of male germ cells is the key factor influencing delivery of fatherly genetic material to an ovum does not raise doubts. Kinetic characteristics define quality, viability of spermatozoids and its cryotolerance. Decrease of spermatozoids mobility can lead to an astenozoospermia and be the reason of male infertility. Correction of spermatogenesis is most often carried out by use of hormonal therapy which possesses side effect breaking physiological parameters of an organism. The low efficiency of therapy is caused by insufficiency of knowledge of the factors defining mobility of gametes. In this regard the great interest is attracted by drugs on the basis of peloid. Components of peloid have antioxidant, reparative, cytoprotective, proliferative effect. Maintaining constancy of structure of bio-systems, humic substances (HS) of peloid promote restoration of biological norm at disease states.

The aim of this work was a research of impact of HS of peloid on mobility of men's gametes.

The experiment was conducted on volunteers at the age of $25-30$. Fractions of $0.1 \%$ solutions of humic substances of peloids were used: humic acids (HA), hymatomelanic acids (HMA), and initial HS extract (HFA). In the series of experiments a buffer solution FertiCult flushing (HEPES, $4 \mathrm{~g} / \mathrm{L}$ HSA) (FertiPro, Belgium) and a specimen solution were added to the target volume of the purified ejaculation sample. A series of experiments with the different content of peloid drugs was carried out to determine the optimal concentration. Changes in sperm motility were observed after 10, 30, 60, 120 and 240 minutes of incubation at room temperature. Ejaculation analysis was carried out using Nikon Eclipse E200 microscope (Japan) and computer sperm analysis Sperm Class Analyzer from Microptic SL (Barcelona, Spain) in accordance with WHO recommendations. The results were evaluated using the statistical software package SPSS 12.0.2 of the same name company (or Statistica 6.0 of Statsoft). Based on the results of data allocation checking for compliance with the normal allocation, nonparametric methods of comparing the parameters in the test groups (Mann-Whitney criterion) were used. The data obtained by us prove adaptogenic properties of humic peloid drugs, which do not have a negative impact on the life of biotest. We have identified differences in the kinetic characteristics of the tests depending on the type of drug. The results of sperm motility measuring in samples with the addition of HS show that in 10 minutes of incubation there is an improvement in the kinetic properties of sperm.

Thus, the humic components of the peloids have the ability to increase human sperm motility in vitro. The intensity of the effect depends on the type of peloid drug, concentration, incubation time. The obtained results determine the perspective of studies of the effect of humic peloid drugs on male fertility function. 